\title{
Microwave-Promoted Synthesis of Novel $N$-Arylanthranilic Acids
}

\author{
Mauro A. Bueno, Larissa R. S. P. Silva and Arlene G. Corrêa* \\ Departamento de Química, Universidade Federal de São Carlos, 13565-905 São Carlos-SP, Brazil
}

\begin{abstract}
Nesse trabalho é relatada a síntese de uma série de ácidos $N$-aril antranílicos inéditos, com bons a excelentes rendimentos, empregando irradiação de microondas como fonte de aquecimento para promover a reação de acoplamento de Ullmann entre ácidos antranílicos e brometos de arila contendo grupos substituintes doadores ou aceptores de elétrons.
\end{abstract}

In this paper we report the synthesis of a series of novel $\mathrm{N}$-aryl anthranilic acids, with good to excellent yields, employing microwaves as heat source to promote the Ullmann coupling between anthranilic acids and aryl bromides possessing electron donating or withdrawing groups.

Keywords: $N$-aryl anthranilic acids, microwaves, Ullmann coupling, green chemistry

\section{Introduction}

$N$-Aryl anthranilic acids are an interesting class of compounds which shows antibacterial ${ }^{1,2}$ and antiinflammatory ${ }^{3}$ properties. It is also employed as precursor of biologically important compounds such as acridine $e^{4-6}$ and acridone alkaloids. ${ }^{7-14}$ The synthesis of $\mathrm{N}$-aryl anthranilic acids can be usually achieved by the Ullmann condensation ${ }^{15}$ between a 2-halobenzoic acid and an aryl amine ${ }^{16,17}$ or an anthranilic acid and an aryl halide. ${ }^{18,19}$ One of the major drawback of these methodologies is the need of heating during long reaction time. ${ }^{20}$

One of the twelve principles of Green Chemistry is that energy requirements of chemical processes should be recognized for their environmental and economic impacts and should be minimized. ${ }^{21}$ The use of unconventional energy sources, as for example ultrasound or microwave (MW) irradiation, may greatly reduce energy consumption. Methods for carried out Ullmann reaction under ultrasonic irradiation have been reported. ${ }^{22,23}$

The use of microwaves in organic synthesis has grown in recent years and among its advantages, when compared to the reactions carried out in conventional heating, are the reduction of reaction times and, in many cases, a significant increase in yields of desired products. ${ }^{24-27}$ There are few reports in literature describing the Ullmann coupling employing microwave heating using water as solvent ${ }^{28,29}$ and solvent-free conditions. ${ }^{30}$

*e-mail: agcorrea@power.ufscar.br
In this paper we report the synthesis of a series of novel $N$-aryl anthranilic acids, with good to excellent yields, employing microwaves as heat source to promote the Ullmann coupling between anthranilic acids and aryl bromides possessing electron donating or withdrawing groups.

\section{Results and Discussion}

In an ongoing program on the synthesis of acridone alkaloids, we firstly investigated the Ullmann reaction of 1-benzyloxy-3-bromobenzene (1a) and an anthranilic acid (2). After a series of experiments employing several literature available procedures, ${ }^{31-34}$ we observed that this reaction was best performed using the methodology described by Ma and co-workers ${ }^{20}$ (Scheme 1), employing $20 \mathrm{~mol} \%$ of $\mathrm{Cu}$ and L-proline, DMSO as solvent at $90{ }^{\circ} \mathrm{C}$ for $24 \mathrm{~h}$.

Our attempts to obtain the desired compounds under analogous conditions in a focused microwave oven $\left(\right.$ CEM-Discover ${ }^{\circledR}$ ) were unsuccessful (Table 1, entry 1). The reason for this lack of reactivity may be due to the high dielectric loss of dimethylsulfoxide which makes the microwave effect less pronounced. ${ }^{26,35}$ Then, we performed the reaction between compound $\mathbf{1 a}$ and $\mathbf{2 a}$ with the same catalytic system but using iso-amyl alcohol as solvent and after one hour the desired compound was isolated in $78 \%$ yield (entry 2). However, reaction between aryl halide 1a and anthranilic acid $\mathbf{2 b}$ in analogous conditions furnished no product. The low solubility of $\mathbf{2} \mathbf{b}$ in iso-amyl alcohol 
<smiles>Brc1cccc(OCc2ccccc2)c1</smiles>

$1 \mathrm{a}$<smiles>[R]c1ccc(N)c(C(=O)O)c1</smiles>

2a $\mathrm{R}=\mathrm{H}$

2b $\mathrm{R}=\mathrm{NO}_{2}$
Cul, L-Proline, $\mathrm{K}_{2} \mathrm{CO}_{3}$ DMSO, $90^{\circ} \mathrm{C}, 24 \mathrm{~h}$<smiles>[R]c1ccc(Nc2cccc(OCc3ccccc3)c2)c(C(=O)O)c1</smiles>

$3 a \mathrm{R}=\mathrm{H} \quad 69 \%$ $3 \mathrm{~b} \mathrm{R}=\mathrm{NO}_{2} \quad 77 \%$

Scheme 1. Synthesis of $N$-arylanthranilic acids.

seemed to be responsible for the unsatisfactory result, thus we performed the reaction of $\mathbf{1 a}$ and $\mathbf{2} \mathbf{b}$ in anhydrous DMF and compound $\mathbf{3 b}$ was obtained in $59 \%$ yield. The yield of $\mathbf{3 b}$ was improved to $82 \%$ by the use of DMF containing $10 \%$ of water (entries 6-8).

Water effect was also observed in the reaction of anthranilic acid $\mathbf{2 b}$ with $p$-bromoanisol (1b). In anhydrous DMF, compound $\mathbf{3 c}$ was obtained in $39 \%$ while it was isolated in $76 \%$ when performed in DMF/water (entries 9 and 10). Reaction of $\mathbf{1 a}$ and $\mathbf{2 a}$ in DMF/water resulted in the formation of a complex mixture of by-products; additionally use of $i s o$-amyl alcohol/water resulted in the recovery of starting materials (entries 4 and 5). Formation of by-products in DMF/water system may be attributed to the higher reaction temperature. By the other hand, the lack of reactivity in iso-amyl alcohol/water system may be due to the lower solubility of the reagents, however this effect is not clear yet since the solvent dramatically affect the Ullmann reaction and inhibition of the cooper catalytic species should also be considered. ${ }^{36}$

After optimization of the Ullmann reaction we performed the coupling of different anthranilic acids and aryl halides possessing electron donating and electron withdrawing groups to evaluate the scope of this protocol
(Table 2). Good to excellent isolated yields were obtained in all cases, excepting with difluor-substitute anthranilic acid 2f, which afforded relatively low yield probably due to its low reactivity once starting material was recovered.

\section{Conclusions}

In summary, we have demonstrated that Ullmann coupling reaction between anthranilic acids and aryl bromides employing microwave heating is an advantage method since desired products can be obtained in good yields and shorter reaction time when compared to the conventional heating. Employing this protocol we prepared a series of novel $\mathrm{N}$-arylanthranilic acids with good to excellent yields.

\section{Experimental}

Unless otherwise noted, all commercially available reagents were purchased from Aldrich Chemical Co. Reagents and solvents were purified when necessary according to the usual procedures described in the literature. ${ }^{1} \mathrm{H}$ and ${ }^{13} \mathrm{C}$ NMR spectra were recorded on a Bruker ARX-200 (200 and $50 \mathrm{MHz}$ respectively). The IR spectra refer to films and were measured on a Bomem M102

Table 1. Ullmann coupling employing microwave irradiation

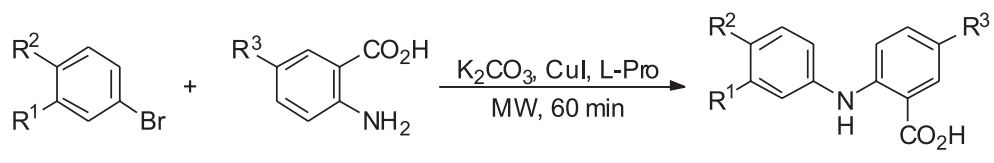

1

2

3

\begin{tabular}{|c|c|c|c|c|c|}
\hline Entry & Aryl bromide & Anthranilic acid & Solvent & Temperature / $\left({ }^{\circ} \mathrm{C}\right)$ & Product, yield / $(\%)^{\mathrm{a}}$ \\
\hline 1 & 1a: $\mathrm{R}^{1}=\mathrm{BnO} ; \mathrm{R}^{2}=\mathrm{H}$ & 2a: $\mathrm{R}^{3}=\mathrm{H}$ & DMSO & 90 & $\mathrm{NR}$ \\
\hline 2 & $1 \mathrm{a}$ & $\mathbf{2 a}$ & iso-amyl alcohol & 140 & 3a: $\mathrm{R}^{1}=\mathrm{BnO}, \mathrm{R}^{2}=\mathrm{R}^{3}=\mathrm{H}(78 \%)$ \\
\hline 3 & $1 \mathbf{a}$ & $2 \mathbf{a}$ & DMF & 160 & NR \\
\hline 4 & $1 \mathbf{a}$ & $\mathbf{2 a}$ & iso-amyl alcohol/ $\mathrm{H}_{2} \mathrm{O}$ & 140 & NR \\
\hline 5 & $1 \mathbf{a}$ & $\mathbf{2 a}$ & $\mathrm{DMF} / \mathrm{H}_{2} \mathrm{O}$ & 160 & NR \\
\hline 6 & $1 \mathbf{a}$ & $\mathbf{2 b}: \mathrm{R}^{3}=\mathrm{NO}_{2}$ & iso-amyl alcohol & 140 & $\mathrm{NR}$ \\
\hline 7 & $1 \mathbf{a}$ & $2 \mathbf{b}$ & $\mathrm{DMF}$ & 160 & 3b: $\mathrm{R}^{1}=\mathrm{BnO} ; \mathrm{R}^{2}=\mathrm{H} ; \mathrm{R}^{3}=\mathrm{NO}_{2}(59 \%)$ \\
\hline 8 & $1 \mathbf{a}$ & $2 \mathbf{b}$ & $\mathrm{DMF} / \mathrm{H}_{2} \mathrm{O}$ & 160 & $\mathbf{3 b}(82 \%)$ \\
\hline 9 & 1b: $\mathrm{R}^{1}=\mathrm{H} ; \mathrm{R}^{2}=\mathrm{MeO}$ & $2 \mathbf{b}$ & DMF & 160 & 3c: $\mathrm{R}^{1}=\mathrm{H} ; \mathrm{R}^{2}=\mathrm{MeO} ; \mathrm{R}^{3}=\mathrm{NO}_{2}(39 \%)$ \\
\hline 10 & 1b & $2 \mathbf{b}$ & $\mathrm{DMF} / \mathrm{H}_{2} \mathrm{O}$ & 160 & $3 \mathbf{c}(76 \%)$ \\
\hline
\end{tabular}

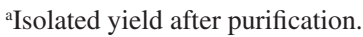


Table 2. Ullmann reaction of different anthranilic acids and aryl halides ${ }^{\mathrm{a}}$

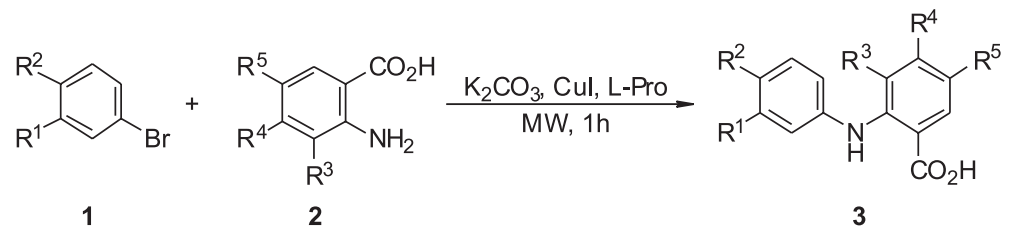

\begin{tabular}{|c|c|c|c|}
\hline Entry & Aryl bromide & Anthranilic acid & Product, yield / $(\%)^{\mathrm{b}}$ \\
\hline 1 & 1c: $\mathrm{R}^{1}=\mathrm{NO}_{2} ; \mathrm{R}^{2}=\mathrm{BnO}$ & $\mathbf{2 a}: \mathrm{R}^{3}=\mathrm{R}^{4}=\mathrm{R}^{5}=\mathrm{H}$ & 3d, 83 \\
\hline 2 & 1d: $\mathrm{R}^{1}=\mathrm{MeO} ; \mathrm{R}^{2}=\mathrm{BnO}$ & $\mathbf{2 a}$ & $3 e, 85$ \\
\hline 3 & 1e: $\mathrm{R}^{1}=\mathrm{H} ; \mathrm{R}^{2}=\mathrm{BnO}$ & 2b: $\mathrm{R}^{3}=\mathrm{R}^{4}=\mathrm{H} ; \mathrm{R}^{5}=\mathrm{NO}_{2}$ & 3f, 69 \\
\hline 4 & 1f: $\mathrm{R}^{1} ; \mathrm{R}^{2}=\mathrm{H}$ & $2 b$ & $3 g, 82$ \\
\hline 5 & $1 \mathrm{c}$ & & 3h, 54 \\
\hline 6 & 1d & $\mathrm{NH}_{2}$ & $3 \mathbf{i}, 81$ \\
\hline 7 & 1c & 2d: $\mathrm{R}^{3}=\mathrm{MeO} ; \mathrm{R}^{4}=\mathrm{R}^{5}=\mathrm{H}$ & $\mathbf{3 j}, 92$ \\
\hline 8 & 1d & $2 d$ & $\mathbf{3 k}, 70$ \\
\hline 9 & 1c & 2e: $\mathrm{R}^{3}=\mathrm{R}^{4}=\mathrm{R}^{5}=\mathrm{MeO}$ & 31, 83 \\
\hline 10 & 1d & 2f: $\mathrm{R}^{3}=\mathrm{H} ; \mathrm{R}^{4}=\mathrm{R}^{5}=\mathrm{F}$ & $\mathbf{3 m}, 38$ \\
\hline 11 & 1c & $2 f$ & $3 n, 54$ \\
\hline
\end{tabular}

${ }^{\mathrm{a}} \mathrm{All}$ reactions were performed in anhydrous iso-amyl alcohol as solvent at $140{ }^{\circ} \mathrm{C}$, except for anthranilic acid $\mathbf{2} \mathbf{b}$ which was performed in $\mathrm{DMF} / \mathrm{H}_{2} \mathrm{O}$ $90 \%$ at $160{ }^{\circ} \mathrm{C}$. ${ }^{\mathrm{b}}$ Isolated yield after purification.

spectrometer. Mass Spectra were recorded on a Shimadzu GCMS-QP5000 or Mass Spectrometer QuatroLCMicromass. Elemental analyses were performed on a Fisons EA 1108 CHNS-O. Analytical thin-layer chromatography was performed on a $0.25 \mu \mathrm{m}$ film of silica gel containing fluorescent indicator $\mathrm{UV}_{254}$ supported on an aluminum sheet (Sigma-Aldrich). Flash column chromatography was performed using silica gel (Kieselgel 60, 230-400 mesh, E. Merck). Gas chromatography was performed in a Shimadzu GC-17A with $\mathrm{H}_{2}$ as carrier and using a DB-5 column. Melting points were performed in Microquimica MQAPF-301.

\section{General procedure}

Aryl bromide ( $0.84 \mathrm{mmol})$, anthranilic acid $(0.42 \mathrm{mmol})$, $\mathrm{CuI}(0.084 \mathrm{mmol})$, L-proline $(0.084 \mathrm{mmol}), \mathrm{K}_{2} \mathrm{CO}_{3}$ $(0.42 \mathrm{mmol})$ and solvent $(1 \mathrm{~mL})$ (Tables 1 and 2$)$ were placed in a glass tube, purged with oxygen-free nitrogen during $10 \mathrm{~min}$ sealed and irradiated during $1 \mathrm{~h}$ in a focused microwave oven (CEM Discover®).

\section{2-(3-Benzyloxy-phenylamino)-benzoic acid $(3 \boldsymbol{a})^{37}$}

Purified by flash chromatography employing hexanes: ethyl acetate:acetic acid (90:10:0.3) as eluent; white solid; mp $134.8^{\circ} \mathrm{C}$; ${ }^{1} \mathrm{H}$ NMR $\left(200 \mathrm{MHz}, \mathrm{CDCl}_{3}\right): \delta 5.11(\mathrm{~s}, 1 \mathrm{H})$, 6.96-6.76 (m, 4H), 7.51-7.24 (m, 8H), $8.10\left(\mathrm{dd}, J_{1} 8.1,1.4\right.$ $\mathrm{Hz}, 1 \mathrm{H}), 9.35(\mathrm{~s}, 1 \mathrm{H}) .{ }^{13} \mathrm{C} \mathrm{NMR}\left(50 \mathrm{MHz}, \mathrm{CDCl}_{3}\right): \delta 70.0$, 109.3, 110.4, 110.6, 114.4, 115.3, 117.3, 127.4 (2C), 127.9, 128.5 (2C), 130.1, 132.5, 135.2, 136.8, 141.6, 148.5, 159.7,
173.8. Anal. Calc. for $\mathrm{C}_{20} \mathrm{H}_{17} \mathrm{NO}_{3}$ : C 75.22; $\mathrm{H}$ 5.37; $\mathrm{N} 4.39$. Found: C 75.02; H 5.40; N 5.20.

\section{2-(3-Benzyloxy-phenylamino)-5-nitrobenzoic acid (3b)}

Purified by flash chromatography employing hexanes: ethyl acetate:acetic acid (70:30:0.3) as eluent; yellow solid; mp $194.3{ }^{\circ} \mathrm{C}$ (dec.); ${ }^{1} \mathrm{H}$ NMR (200 MHz, $\left.\mathrm{CDCl}_{3}\right): \delta 5.07$ (s, 2H), 6.94-6.77 (m, 1H), 7.06 (d, J 9.4 Hz, 1H), 7.38-7.21 $(\mathrm{m}, 7 \mathrm{H}), 8.06(\mathrm{dd}, J 9.4,2.7 \mathrm{~Hz}, 1 \mathrm{H}), 8.93(\mathrm{~d}, J 2.7 \mathrm{~Hz}, 1 \mathrm{H})$, $10.42(\mathrm{~s}, 1 \mathrm{H}) .{ }^{13} \mathrm{C} \mathrm{NMR}\left(50 \mathrm{MHz}, \mathrm{CDCl}_{3}\right): \delta 69.8,76.3,77.0$, 77.6, 110.4, 110.8, 112.1, 112.7, 16.3, 127.1 (2C), 127.8, 128.4 (2C), 128.8, 129.2, 130.2, 136.3, 137.0, 139.5, 152.5, 159.5, 169.3. Anal. Calc. for $\mathrm{C}_{20} \mathrm{H}_{16} \mathrm{~N}_{2} \mathrm{O}_{5}$ : C 65.93; $\mathrm{H} 4.43$; N 7.69. Found: C 65.13; H 4.50; N 7.03.

\section{2-(4-Methoxy-phenylamino)-5-nitrobenzoic acid (3c)}

Purified by flash chromatography employing hexanes: ethyl acetate:acetic acid (70:30:0.3) as eluent; yellow solid; mp $230.8{ }^{\circ} \mathrm{C} ;{ }^{1} \mathrm{H}$ NMR (200 MHz, DMSO- $\left.d_{6}\right): \delta 3.77$ $(\mathrm{s}, 3 \mathrm{H}), 6.84-7.27(\mathrm{~m}, 6 \mathrm{H}), 8.08(\mathrm{dd}, J 9.0,4.0 \mathrm{~Hz}, 1 \mathrm{H})$, $8.66(\mathrm{~d}, J 4.0 \mathrm{~Hz}, 2 \mathrm{H}), 10.71(\mathrm{~s}, 1 \mathrm{H}) .{ }^{13} \mathrm{C} \mathrm{NMR}(50 \mathrm{MHz}$, DMSO- $\left.d_{6}\right): \delta 55.2,110.1,112.7,114.9$ (2C), 126.4 (2C), 128.4, 129.1, 130.6, 136.0, 137.0, 153.4, 157.5, 168.6. Anal. Calc. for $\mathrm{C}_{14} \mathrm{H}_{12} \mathrm{~N}_{2} \mathrm{O}_{5}$ : C 58.33; H 4.20; N 9.72. Found: C 58.09; H 4.33; N 9.72.

\section{2-(4-Benzyloxy-3-nitro-phenylamino)-benzoic acid (3d)}

Purified by flash chromatography employing hexanes: ethyl acetate:acetic acid (70:30:0.3) as eluent; yellow solid; mp $197.2{ }^{\circ} \mathrm{C} ;{ }^{1} \mathrm{H}$ NMR (200 MHz, DMSO- $d_{6}$ ): $\delta$ 
$5.28(\mathrm{~s}, 2 \mathrm{H}), 6.80(\mathrm{t}, J 8.0 \mathrm{~Hz}, 1 \mathrm{H}), 7.09(\mathrm{~d}, J 8.0 \mathrm{~Hz}, 1 \mathrm{H})$, 7.63-7.56 (m, 9H), $7.77(\mathrm{~d}, J 2.0 \mathrm{~Hz}, 1 \mathrm{H}), 7.90(\mathrm{~d}, J 8.0$ $\mathrm{Hz}, 1 \mathrm{H}), 9.55(\mathrm{~s}, 1 \mathrm{H}) .{ }^{13} \mathrm{C}$ NMR $\left(50 \mathrm{MHz}, \mathrm{DMSO}-d_{6}\right): \delta$ 70.7, 104.7, 112.9, 113.6, 116.8, 117.8, 118.2, 127.3 (2C), 128.0, 128.1, 128.4 (2C), 131.8, 133.8, 134.2, 136.0, 139.9, 146.8, 168.6. Anal. Calc. for $\mathrm{C}_{20} \mathrm{H}_{16} \mathrm{~N}_{2} \mathrm{O}_{5}$ : C 65.93; $\mathrm{H} 4.43$; N 7.69. Found: C 66.20; H 4.70; N 8.11.

2-(4-Benzyloxy-3-methoxy-phenylamino)-benzoic acid (3e)

Purified by flash chromatography employing hexanes: ethyl acetate:acetic acid (80:20:0.3) as eluent; yellowish solid; mp $182.5{ }^{\circ} \mathrm{C} ;{ }^{1} \mathrm{H}$ NMR (200 MHz, DMSO- $\left.d_{6}\right): \delta$ 3.76 (s, 3H), 5.05 (s, 2H), 6.65-6.87 (m, 2H), 6.99 (d, $J$ $2.0 \mathrm{~Hz}, 2 \mathrm{H}), 7.28-7.46(\mathrm{~m}, 7 \mathrm{H}), 7.86(\mathrm{dd}, J 8.0,2.0 \mathrm{~Hz}$, $1 \mathrm{H}), 9.45(\mathrm{~s}, 1 \mathrm{H}) .{ }^{13} \mathrm{C} \mathrm{NMR}\left(50 \mathrm{MHz}, \mathrm{DMSO}-d_{6}\right): \delta 55.7$, 70.3, 108.3, 111.4, 113.1, 114.6, 114.8, 116.3, 127.6 (2C), 127.7, 128.2 (2C), 131.6, 133.8, 134.1, 137.2, 144.5, 148.3, 149.9, 169.9. Anal. Calc. for $\mathrm{C}_{21} \mathrm{H}_{19} \mathrm{NO}_{4}$ : C 72.19; $\mathrm{H}$ 5.48; N 4.01. Found: C 71.94; H 5.92; N 4.44.

\section{2-(4-Benzyloxy-phenylamino)-5-nitrobenzoic acid (3f)}

Purified by flash chromatography employing hexanes: ethyl acetate:acetic acid (60:40:0.3) as eluent; yellow solid; mp $202.5{ }^{\circ} \mathrm{C} ;{ }^{1} \mathrm{H}$ NMR (200 MHz, DMSO- $\left.d_{6}\right): \delta$ $5.12(\mathrm{~s}, 2 \mathrm{H}), 6.92-7.44(\mathrm{~m}, 13 \mathrm{H}), 8.10(\mathrm{dd}, J$ 10.0, 4.0 $\mathrm{Hz}, 1 \mathrm{H}), 8.70$ (d, J 4.0 Hz, 1H), 10.42 (s, 1H). ${ }^{13} \mathrm{C}$ NMR (50 MHz, DMSO- $\left.d_{6}\right): \delta 69.4,111.2,112.6,115.8$ (2C), 126.2 (2C), 127.6 (2C), 127.8, 128.3 (2C), 128.4, 138.9, 131.0, 135.9, 136.8, 153.3, 156.4, 168.7. Anal. Calc. for $\mathrm{C}_{20} \mathrm{H}_{16} \mathrm{~N}_{2} \mathrm{O}_{5}$ : C 65.93; H 4.43; N 7.69. Found: C 66.22; $\mathrm{H} 4.75$; N 7.94 .

\section{5-Nitro-2-phenylaminobenzoic acid (3g)}

Purified by flash chromatography employing hexanes: ethyl acetate:acetic acid (70:30:0.3) as eluent; yellow solid; mp $241.3{ }^{\circ} \mathrm{C}$ (dec.); ${ }^{1} \mathrm{H}$ NMR (200 MHz, DMSO- $\left.d_{6}\right): \delta 7.09-$ $7.50(\mathrm{~m}, 7 \mathrm{H}), 8.15(\mathrm{dd}, J 8.0,2.0 \mathrm{~Hz}, 1 \mathrm{H}), 8.69(\mathrm{~d}, J 2.0 \mathrm{~Hz}$, $1 \mathrm{H}), 10.36(\mathrm{~s}, 1 \mathrm{H}) .{ }^{13} \mathrm{C} \mathrm{NMR}\left(50 \mathrm{MHz}, \mathrm{DMSO}-d_{6}\right): \delta 110.9$, 113.1, 123.9 (2C), 125.7, 128.3, 129.2, 129.7 (2C), 136.5, 138.1, 152.3, 168.5. Anal. Calc. for $\mathrm{C}_{13} \mathrm{H}_{10} \mathrm{~N}_{2} \mathrm{O}_{4}: \mathrm{C} 60.47$; H 3.90; N 10.85. Found: C 60.59; H 4.02; N 10.89 .

3-(4-Benzyloxy-3-nitro-phenylamino)-2-naphthoic acid (3h)

Purified by flash chromatography employing hexanes: ethyl acetate:acetic acid (70:30:0.3) as eluent; yellow solid; mp $209.5{ }^{\circ} \mathrm{C}$ (dec.) $;{ }^{1} \mathrm{H}$ NMR (200 MHz, DMSO- $d_{6}$ ): $\delta 5.05$ (s, 2H), 7.26-7.29 (m, 14H), $8.64(\mathrm{~s}, 1 \mathrm{H}), 9.38(\mathrm{~s}, 1 \mathrm{H}) .{ }^{13} \mathrm{C}$ NMR $\left(50 \mathrm{MHz}, \mathrm{DMSO}-d_{6}\right): \delta 70.8,99.5,103.5,108.0$, 116.4, 116.9, 117.4, 123.4, 125.7, 126.2, 127.4 (2C), 128.0,
128.4 (2C), 128.8, 133.7, 134.6, 136.1, 136.3, 140.1, 142.2, 146.4, 169.4. Anal. Calc. for $\mathrm{C}_{24} \mathrm{H}_{18} \mathrm{~N}_{2} \mathrm{O}_{5}: \mathrm{C} 69.56 ; \mathrm{H} 4.38$; N 6.76. Found: C 69.73; H 4.61; N 6.92.

\section{3-(4-Benzyloxy-3-methoxy-phenylamino)-2-naphthoic acid (3i)}

Purified by flash chromatography employing hexanes: ethyl acetate:acetic acid (70:30:0.3) as eluent; yellow solid; mp $198.9{ }^{\circ} \mathrm{C}$ (dec.); ${ }^{1} \mathrm{H}$ NMR (200 MHz, DMSO- $\left.d_{6}\right): \delta$ $3.80(\mathrm{~s}, 3 \mathrm{H}), 5.09(\mathrm{~s}, 2 \mathrm{H}), 6.85-7.08(\mathrm{~m}, 3 \mathrm{H}), 7.19-7.63(\mathrm{~m}$, $10 \mathrm{H}), 7.86$ (d, $J 10.0 \mathrm{~Hz}, 1 \mathrm{H}), 8.62(\mathrm{~s}, 1 \mathrm{H}) .{ }^{13} \mathrm{C}$ NMR $(50$ MHz, DMSO- $\left.d_{6}\right): \delta 55.8,70.4,99.58,106.7,107.7,114.1$, $114.8,115.4,122.7,125.5,125.5,127.7$ (2C), 128.3 (2C), 128.7, 128.9, 133.6, 134.5, 136.6, 137.3, 143.8, 144.1, 150.0, 169.7. Anal. Calc. for $\mathrm{C}_{25} \mathrm{H}_{21} \mathrm{NO}_{4}$ : C 75.17; H 5.30; N 3.51. Found: C 74.90; H 5.15; N 3.73.

2-(4-Benzyloxy-3-nitro-phenylamino)-3-methoxybenzoic acid (3j)

Purified by flash chromatography employing hexanes: ethyl acetate:acetic acid (75:25:0.3) as eluent; yellow solid; mp $178.1{ }^{\circ} \mathrm{C}$; ${ }^{1} \mathrm{H}$ NMR (200 MHz, DMSO- $\left.d_{6}\right): \delta 3.73$ (s, $3 \mathrm{H}), 5.21(\mathrm{~s}, 2 \mathrm{H}), 6.98-7.50(\mathrm{~m}, 12 \mathrm{H}), 8.69(\mathrm{~s}, 1 \mathrm{H}) .{ }^{13} \mathrm{C}$ NMR $\left(50 \mathrm{MHz}, \mathrm{DMSO}-d_{6}\right): \delta 55.5,71.0,111.9,116.1$, $116.3,122.1,122.3,122.5,122.8,127.4$ (2C), 127.9, 128.4 (2C), 132.4, 136.4 138.3, 139.5, 144.3, 152.2, 169.0. Anal. Calc. for $\mathrm{C}_{21} \mathrm{H}_{18} \mathrm{~N}_{2} \mathrm{O}_{5}$ : C 63.96; $\mathrm{H} 4.60 ; \mathrm{N} 7.10$. Found: $\mathrm{C}$ 64.32; H 4.95; N 7.49.

2-(4-Benzyloxy-3-methoxy-phenylamino)-3-methoxybenzoic acid $(3 k)$

Purified by flash chromatography employing hexanes: ethyl acetate:acetic acid (80:20:0.3) as eluent; yellowish solid; mp $187.6{ }^{\circ} \mathrm{C} ;{ }^{1} \mathrm{H}$ NMR (200 MHz, DMSO- $\left.d_{6}\right)$ : $\delta 3.67$ $(\mathrm{s}, 3 \mathrm{H}), 3.68(\mathrm{~s}, 3 \mathrm{H}), 4.95(\mathrm{~s}, 2 \mathrm{H}), 6.15(\mathrm{dd}, J 8.0,2.0 \mathrm{~Hz}, 1 \mathrm{H})$, $6.43(\mathrm{~d}, J 2.0 \mathrm{~Hz}, 1 \mathrm{H}), 6.80(\mathrm{~d}, J 8.0 \mathrm{~Hz}, 1 \mathrm{H}), 7.01(\mathrm{t}, J 8.0$ $\mathrm{Hz}, 1 \mathrm{H}), 7.19-7.46(\mathrm{~m}, 8 \mathrm{H}) .{ }^{13} \mathrm{C} \mathrm{NMR}\left(50 \mathrm{MHz}, \mathrm{DMSO}-d_{6}\right)$ : $\delta$ 55.4 (2C), 70.8, 103.1, 108.6, 114.8, 116.0, 120.6, 121.0, 122.7, 127.6 (2C), 127.6, 128.2 (2C), 134.5, 137.5, 138.8, 141.8, 149.4, 151.8, 169.4. Anal. Calc. for $\mathrm{C}_{22} \mathrm{H}_{21} \mathrm{NO}_{5}: \mathrm{C}$ 69.64; H 5.58; N 3.69. Found: C 69.26; H 6.09; N 3.85.

\section{2-(4-Benzyloxy-3-nitro-phenylamino)-3,4,5-trimethoxy- benzoic acid $(3 \mathrm{l})$}

Purified by flash chromatography employing hexanes: ethyl acetate:acetic acid (70:30:0.3) as eluent; yellow solid; mp $187.7{ }^{\circ} \mathrm{C}$; ${ }^{1} \mathrm{H}$ NMR (200 MHz, DMSO- $\left.d_{6}\right): \delta 3.57$ (s, $3 \mathrm{H}), 3.84(\mathrm{~s}, 3 \mathrm{H}), 3.86(\mathrm{~s}, 3 \mathrm{H}), 5.21(\mathrm{~s}, 2 \mathrm{H}), 7.04-7.44(\mathrm{~m}$, $10 \mathrm{H}) .{ }^{13} \mathrm{C}$ NMR $\left(50 \mathrm{MHz}, \mathrm{DMSO}-d_{6}\right): \delta 55.9,59.2,60.7$, 70.9, 109.3, 111.7, 116.5, 117.2, 122.1, 127.3 (2C), 127.9, 128.4 (2C), 131.5, 136.4, 138.9, 139.6, 144.3, 146.5, 146.9, 
148.2, 168.3. Anal. Calc. for $\mathrm{C}_{23} \mathrm{H}_{22} \mathrm{~N}_{2} \mathrm{O}_{8}$ : C 60.79; $\mathrm{H} 4.88$; N 6.16. Found: C 61.14; H 5.13; N 6.46.

2-(4-Benzyloxy-3-methoxy-phenylamino)-4,5-difluorobenzoic acid $(3 m)$

Purified by flash chromatography employing hexanes: ethyl acetate:acetic acid (80:20:0.3) as eluent; white solid; mp $192.7{ }^{\circ} \mathrm{C}$; ${ }^{1} \mathrm{H}$ NMR $\left(200 \mathrm{MHz}, \mathrm{DMSO}-d_{6}\right): \delta 3.76(\mathrm{~s}$, $3 \mathrm{H}), 5.06(\mathrm{~s}, 2 \mathrm{H}), 6.75-7.05(\mathrm{~m}, 5 \mathrm{H}), 7.29-7.48(\mathrm{~m}, 5 \mathrm{H})$, 7.78 (dd, $J 11.0,8.0 \mathrm{~Hz}, 1 \mathrm{H}), 9.45$ (s, 1H). ${ }^{13} \mathrm{C}$ NMR $(50$ MHz, DMSO- $d_{6}$ ): $\delta 55.6,70.2,101.2(\mathrm{~d}, J 21.5 \mathrm{~Hz}), 107.1$ (dd, $J$ 4.7, $2.5 \mathrm{~Hz}), 108.7$ (d, J 0.5 Hz), 114.5, 115.3, 119.5 (d, J 17.5 Hz), 127.7 (2C), 127.7, 128.3 (2C), 133.0, 137.1, 140.5 (dd, $J 234.0, J 13.5 \mathrm{~Hz}$ ), 145.1, 146.7 (dd, $J$ 10.0, 1.0 $\mathrm{Hz}$ ), 150.0, 153.3 (dd, J 249.2, $13.5 \mathrm{~Hz}$ ), 168.3 (dd, J 2.2, $0.5 \mathrm{~Hz}$ ). Anal. Calc. for $\mathrm{C}_{21} \mathrm{H}_{17} \mathrm{~F}_{2} \mathrm{NO}_{4}: \mathrm{C} 65.45 ; \mathrm{H} 4.45 ; \mathrm{N}$ 3.63. Found: C 65.63; H 5.55; N 3.72.

2-(4-Benzyloxy-3-nitro-phenylamino)-4,5-difluorobenzoic acid (3n)

Purified by flash chromatography employing hexanes: ethyl acetate:acetic acid (80:20:0.3) as eluent; yellow solid; mp $216.8{ }^{\circ} \mathrm{C} ;{ }^{1} \mathrm{H}$ NMR (200 MHz, DMSO- $\left.d_{6}\right): \delta$ 5.06 (s, 2H), 6.97 (dd, J 14.0, 6.0 Hz, 1H), 7.30-7.59 (m, $8 \mathrm{H}), 7.76-7.86$ (m, 2H), 9.55 (s, 1H). ${ }^{13} \mathrm{C}$ NMR (50 MHz, DMSO- $\left.d_{6}\right): \delta 70.2,102.1(\mathrm{~d}, J 20.0 \mathrm{~Hz}), 108.8(\mathrm{dd}, J 4.5$, $2.5 \mathrm{~Hz}), 119.8,119.1,119.7$ (d, J $15.5 \mathrm{~Hz}), 127.7$ (2C), 128.0, 128.4 (2C), 128.7, 133.0, 136.0, 139.9, 141.3 (dd, $J$ 235.7, $13.5 \mathrm{~Hz}), 145.2(\mathrm{dd}, J 9.5,1.0 \mathrm{~Hz}), 147.4,153.3$ (dd, $J 262.0,13.5 \mathrm{~Hz}), 168.1$ (d, $J 1.5 \mathrm{~Hz}$ ). Anal. Calc. for $\mathrm{C}_{20} \mathrm{H}_{14} \mathrm{~F}_{2} \mathrm{~N}_{2} \mathrm{O}_{5}$ : C 60.00; H 3.52; $\mathrm{N} 9.49$; found: C 59.66; $\mathrm{H} 3.87$; N 7.16.

\section{Acknowledgments}

We acknowledge FAPESP and CNPq for financial support.

\section{References}

1. Thorarensen, A.; Li, J.; Wakefield, B. D.; Romero, D. L.; Marotti, K. R.; Sweeney, M, T.; Zurenko, G. E.; Sarver, R. W.; Bioorg. Med. Chem. Lett. 2007, 17, 3113.

2. Thorarensen, A.; Wakefield, B. D.; Romero, D. L.; Marotti, K. R.; Sweeney, M. T.; Zurenko, G. E.; Rohrer, D. C.; Han, F.; Bryant, G. L.; Bioorg. Med. Chem. Lett. 2007, 17, 2823.

3. Bajaj, S.; Sambi, S. S.; Madan, A. K.; Bioorg. Med. Chem. 2004, 12, 3695 .

4. Girault, S.; Grellier, P.; Berecibar, A.; Maes, L.; Mouray, E.; Lemiere, P.; Debreu, M.-A.; Davioud-Charvet, E.; Sergheraert, C.; J. Med. Chem. 2000, 43, 2646.
5. Demeunynck, M.; Charmantray, F.; Martelli, A.; Curr. Pharm. Des. 2001, 7, 1703.

6. Brana, M. F.; Cacho, M.; De Pascual-Teresa, B.; Ramos, A.; Curr. Pharm. Des. 2001, 7, 1745.

7. Boumendjel, A.; Macalou, S.; Ahmed-Belkacem, A.; Blanca, M.; Di Pietro, A.; Bioorg. Med. Chem. 2007, 15, 2892.

8. Winter, R. W.; Kelly, J. X.; Smilkstein, M. J.; Dodean, R.; Bagby, G. C.; Rathbun, R. K.; Levin, J. I.; Hinrichs, D.; Riscoe, M. K.; Exp. Parasitol. 2006, 114, 47.

9. Stefaska, B.; Bontemps-Gracz, M. M.; Antonini, I.; Martelli, S.; Arciemiuk, M.; Piwkowska, A.; Rogacka, D.; Borowski, E.; Bioorg. Med. Chem. 2005, 13, 1969.

10. Kamal, A.; Srinivas, O.; Ramulu, P.; Ramesh, G.; Kumar, P. P.; Bioorg. Med. Chem. Lett. 2004, 14, 4107.

11. Mai, H. D. T.; Gaslonde, T.; Michel, S.; Tillequin, F.; Koch, M.; Bongui, J. B.; Elomri, A.; Seguin, E.; Pfeiffer, B.; Renard, P.; Cordonnier, M. H. C.; Laine, W.; Bailly, C.; Berthier, L. K.; Stéphane Léonce, S.; Hickman, J. A.; Pierre, A.; J. Med. Chem. 2003, 46, 3072.

12. Krishnegowda, G.; Thimmaiah, P.; Hegde, R.; Dass, C.; Houghtonb, P. J.; Thimmaiah, K. N.; Bioorg. Med. Chem. 2002, 10, 2367.

13. Bongui, J. B.; Elomri, A.; Seguin, E.; Tillequin, F.; Pfeiffer, B.; Renard, P.; Pierré, A.; Atassi G.; Chem. Pharm. Bull. 2001, 49, 1077.

14. Su, T. S.; Köhler, B.; Chou, T. C.; Moon Woo Chun, M. W.; Watanabet, K. A.; J. Med. Chem. 1992, 35, 2703.

15. Hassan, J.; Sevignon, M.; Gozzi, C.; Schulz, E.; Lemaire, M.; Chem. Rev. 2002, 102, 1359.

16. Maradolla, M. B.; Amaravathi, M.; Kumar, V. N.; Mouli, G.V. P. C.; J. Mol. Catal. A: Chem. 2007, 266, 47.

17. Mei, X.; August, A. T.; Wolf, C.; J. Org. Chem. 2006, 71, 142.

18. Ozaki, K.; Yamada, Y.; Oine, T.; Ishizuka, T.; Iwasawa, Y.; $J$. Med. Chem. 1985, 28, 568.

19. Rewcastle, G. W.; Denny, W. A.; Synth. Commun. 1987, 17, 309.

20. Zhang, H.; Cai, Q.; Ma, D.; J. Org. Chem. 2005, 70, 5173.

21. Anastas, P. T.; Warner, J. C. Green Chemistry: Theory and Practice. Oxford University Press: New York, 1998.

22. Pellon, R. F.; Docampo, M. L.; Fascio, M. L.; Synth. Commun. 2007, 37, 1853 .

23. Hanoun, J. P.; Galy, J. P.; Tenaglia, A.; Synth. Commun. 1995, $25,2443$.

24. Hayes, B. L.; Aldrichim. Acta 2004, 37, 66.

25. Ferguson, J. D.; Mol. Diversity 2003, 7, 281.

26. Kappe, C. O.; Angew. Chem., Int. Ed. 2004, 43, 6250.

27. Whittaker, A. G.; Mingos, D. M. P.; J. Chem. Soc., Dalton Trans. 2002, 21, 3967.

28. Martín, A.; Pellón, R. F.; Mesa, M.; Docampo, M. L.; Gómes, V.; J. Chem. Res. 2005, 9, 561. 
29. Baqi, Y; Müller, C. E.; Org. Lett. 2007, 9, 1271.

30. Pellón, R. F.; Martín, A. Pu. Y. M.; Ku, Y. Y.; Grieme, H. R.; Bhatia, A. V.; Tetrahedron Lett. 2006, 47, 149.

31. Pu, Y. M.; Ku, Y. Y.; Grieme, H. R.; Bhatia, A. V.; Tetrahedron Lett. 2006, 47, 149.

32. Deng, W.; Wang; Zhang, C.; Liu, L.; Guo, Q. Q. X.; Chin. Chem. Lett. 2006, 17, 313

33. Csuk, R.; Barthel, A.; Raschke, C.; Tetrahedron 2004, 60, 5737.
34. Yamada, Y.; Ozaki, K.; Oine, T.; Ishizuka, T.; Iwasawa, Y.; J. Med. Chem. 1985, 28, 568.

35. Hayes, B. L.; Microwave Synthesis-Chemistry at the Speed of Light, CEM Publishing: USA, 2002.

36. Lindley, J.; Tetrahedron 1984, 40, 1433.

37. Brailey, D. A.; US Patent 4510139 1985. (CA 103:71072)

Received: March 13, 2008 Web Release Date: August 1, 2008

FAPESP helped in meeting the publication costs of this article. 